\title{
Atypical Parathyroid Adenoma in Pregnancy: A Rare Case
}

\author{
Keshava Murthy Suryanarayana ${ }^{1}$, Pindi Veechika
}

\begin{abstract}
Aim and objective: Report a very rare case of atypical parathyroid adenoma in pregnancy presenting as a hypercalcemic crisis, acute pancreatitis, and acute kidney injury and also discuss the issues related to localization of parathyroid adenoma, management of hypercalcemia and its complications, and timing of surgery.

Background: Primary hyperparathyroidism (PHPT) is rare in pregnancy and often unrecognized, as serum calcium is not routinely measured and the physiological changes of pregnancy can mask its diagnosis. In addition, non-specific gastrointestinal symptoms associated with hypercalcemia can mimic that of early pregnancy. Vitamin D deficiency can mask hypercalcemia associated with PHPT.

Case description: A 29-year-old pregnant lady of 23 weeks of gestation presented to the emergency department with an acute abdomen and was diagnosed to have a hypercalcemic crisis associated with acute pancreatitis and acute kidney injury. The evaluation revealed PHPT with parathyroid adenoma. While in hospital she developed complications such as pneumonia, sepsis, anemia, and hypoalbuminemia and was managed by a multidisciplinary team. Subsequently, when her condition was stable, she underwent parathyroidectomy. Histopathology of the specimen revealed atypical parathyroid adenoma. By elective cesarean section at 39 weeks of gestation, she delivered a healthy female baby weighing $2.8 \mathrm{~kg}$. Presently, 1 year after surgery, she has remained eucalcemic.

Conclusion: Hypercalcemia due to PHPT is often unrecognized in pregnancy. Correct diagnosis with localization of adenoma, along with appropriate medical treatment and surgery will improve maternal and fetal outcomes.

Clinical significance: A high index of clinical suspicion is needed for the diagnosis of PHPT in pregnancy. Vitamin D deficiency can mask hypercalcemia associated with PHPT. Effective medical and surgical management will lead to better outcomes.

Keywords: Hypercalcemia, Hyperparathyroidism, Imaging, Parathyroid adenoma, Parathyroid carcinoma, Parathyroid localization, Parathyroidectomy, Postoperative hypocalcemia, Pregnancy.

World Journal of Endocrine Surgery (2021): 10.5005/jp-journals-10002-1313
\end{abstract}

\section{BACKGROUND}

Primary hyperparathyroidism (PHPT) is rare in pregnancy. ${ }^{1}$ The true prevalence of PHPT in pregnancy is unknown because serum calcium is not routinely measured and the physiological changes of pregnancy can mask its diagnosis. Hypoalbuminemia, increased glomerular filtration rate, calcium transport across the placenta and estrogen inhibition of parathyroid hormone (PTH) mediated bone resorption can all result in the appearance of lower serum calcium levels. ${ }^{2}$ Undiagnosed persistent hypercalcemia in pregnancy can lead to increased maternal and fetal morbidity and mortality. Prompt early recognition and treatment improves maternal and fetal outcomes. Parathyroid adenoma is the most common cause of PHPT and atypical parathyroid adenoma and carcinoma can rarely be associated with PHPT. We report a rare case of a primigravida with PHPT, who presented with acute pancreatitis in the second trimester.

\section{Case Description}

A 29-year-old primigravida of 23 weeks of gestation was brought to the hospital emergency department on May 25, 2019, with an acute abdomen. She had acute onset severe pain in the epigastrium radiating to the upper back associated with nausea, vomiting, and decreased urine output. Elsewhere, in the first trimester of this pregnancy, she was detected to have normal serum calcium and low vitamin D, when she had presented with musculoskeletal pains, backache, and constipation and was treated with vitamin D and calcium supplements. There was no other significant past or family history. On physical examination, the patient was conscious and
1,2Department of Endocrinology, MS Ramaiah Medical College,
Bengaluru, Karnataka, India

Corresponding Author: Keshava Murthy Suryanarayana, Department of Endocrinology, MS Ramaiah Medical College, Bengaluru, Karnataka, India, Phone: +91 9742971563, e-mail: dr.suryanarayana@gmail.com

How to cite this article: Suryanarayana KM, Veechika P. Atypical Parathyroid Adenoma in Pregnancy: A Rare Case. World J Endoc Surg 2021;13(1):24-27.

Source of support: Nil

Conflict of interest: None

oriented with temp: $100 \mathrm{~F}$, pulse: $120 /$ minute, BP: $90 / 60 \mathrm{~mm} \mathrm{Hg}$, and RR: 28/minute. There were no skin lesions or palpable neck mass. Abdominal examination revealed diffuse tenderness with guarding, rigidity, and absent bowel sounds and also the uterus height of 23 weeks of gestation. Examination of other systems was normal.

A provisional diagnosis of acute abdomen due to acute pancreatitis was considered and workup for the etiology revealed: serum calcium $-14.4 \mathrm{mg} / \mathrm{dL}$, serum albumin $-2.5 \mathrm{mg} / \mathrm{dL}$, corrected calcium-15.6 mg/dL, serum phosphorus-4.6 mg/dL, serum creatinine $-2.25 \mathrm{mg} / \mathrm{dL}$, vitamin D-52.3 ng/mL, PTH >2,500 $\mathrm{pg} / \mathrm{mL}$, serum amylase-2,920 U/L, serum lipase-1,900 U/L. A diagnosis of PHPT associated with hypercalcemic crisis, acute pancreatitis, and acute kidney injury was considered. MRI of the abdomen confirmed acute pancreatitis. Intravenous hydration with isotonic saline was started, along with injection calcitonin $4 \mathrm{IU} / \mathrm{kg}$ twice daily. Hemodialysis was initiated in view of worsening renal

() The Author(s). 2021 Open Access This article is distributed under the terms of the Creative Commons Attribution 4.0 International License (https://creativecommons. org/licenses/by-nc/4.0/), which permits unrestricted use, distribution, and non-commercial reproduction in any medium, provided you give appropriate credit to the original author(s) and the source, provide a link to the Creative Commons license, and indicate if changes were made. The Creative Commons Public Domain Dedication waiver (http://creativecommons.org/publicdomain/zero/1.0/) applies to the data made available in this article, unless otherwise stated. 
parameters and severe hypercalcemia and serum calcium levels dropped from 15 to $12 \mathrm{mg} / \mathrm{dL}$.

Ultrasound neck revealed a mixed echogenic lesion posterior to the right thyroid gland possibly a parathyroid adenoma (Fig. 1). MRI of the neck and thorax was done for better localization of the lesion before surgery and showed an ill-defined mass in the neck base measuring $19 \times 18 \times 48 \mathrm{~mm}$ on the right side extending to the superior mediastinum with possible encasement of a carotid sheath (Fig. 2).

After discussion with a multidisciplinary team, the patient underwent an elective parathyroid adenoma resection. Intraoperative findings revealed a $4 \times 3 \mathrm{~cm}$ parathyroid tumor, encasing the carotid sheath, adherent to the carotid artery, internal jugular vein, and sternocleidomastoid on the lateral aspects. Serum PTH levels dropped significantly within 5 minutes after resection of adenoma from 1,744 to $248 \mathrm{pg} / \mathrm{mL}$. Postoperatively, serum calcium levels were monitored and PTH levels dropped to $26 \mathrm{pg} / \mathrm{mL}$ on day 1 (Table 1). The patient developed hungry bone syndrome and was treated with IV calcium and active oral vitamin D. Further course was complicated by vocal cord palsy and hospital-acquired pneumonia, which were managed effectively. Serum calcium levels normalized over 1 week period and the patient became stable and was discharged on calcium and vitamin D supplements. Histopathology report was consistent with atypical parathyroid adenoma (Figs 3 and 4). Subsequently, she underwent elective

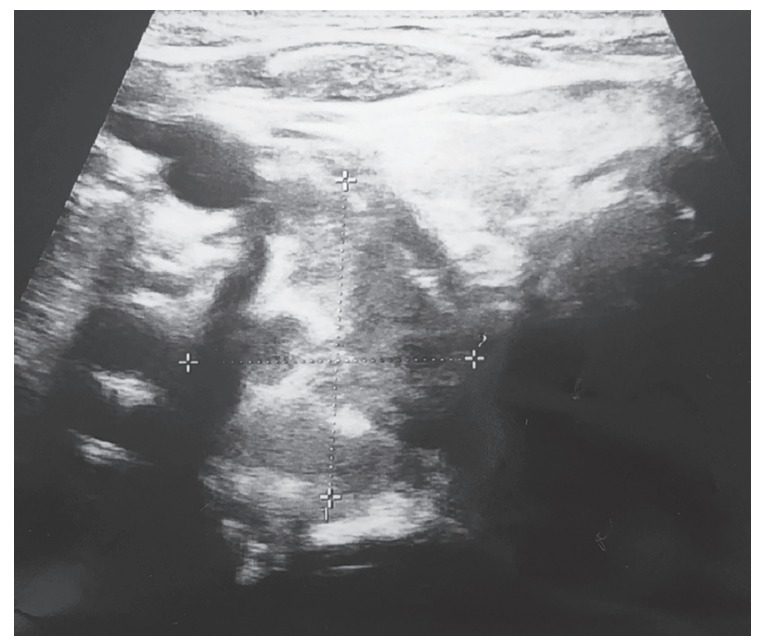

Fig. 1: Ultrasound neck showing ill-defined mixed echogenic lesion posterior to the right of thyroid gland measuring $24 \times 21 \mathrm{~mm}$ cesarean section at 39 weeks of gestation and delivered a healthy female baby with a birth weight of $2.8 \mathrm{~kg}$. On further follow-up at 7 and 12 months after surgery, she has remained euglycemic with mild elevated serum PTH.

\section{Discussion}

Primary hyperparathyroidism is a relatively common disorder affecting 1 in 500 women and 1 in 2,000 men, over 40 years of age. The best estimate of incidence is 22 per 100,000 per year, and prevalence is approximately 1 per $1,000 .^{3}$ In the reproductive age group of 20-40 years in women, the prevalence was found to be $0.05 \%$ in a study where serum calcium levels were measured routinely. ${ }^{4}$ However, as serum calcium levels are not measured routinely in practice, the true prevalence in pregnancy is unknown. Up to $80 \%$ of patients with PHPT in pregnancy are asymptomatic. ${ }^{5}$

Hypercalcemia in pregnancy is associated with significant maternal and neonatal adverse outcomes. Complications as a result of PHPT during pregnancy have been reported to occur in up to $67 \%$ of mothers and $80 \%$ of fetuses. ${ }^{5}$ Maternal complications include hypercalcemic crisis, pancreatitis, nephrolithiasis, hyperemesis, neuropsychiatric symptoms, and muscle weakness. Metabolic bone disease with severe osteoporosis can result in fracture of pelvis and femur neck requiring immobilization. The neonatal complications include intrauterine growth retardation, low birth weight, preterm delivery, intrauterine death, seizures, and neonatal tetany. ${ }^{5}$

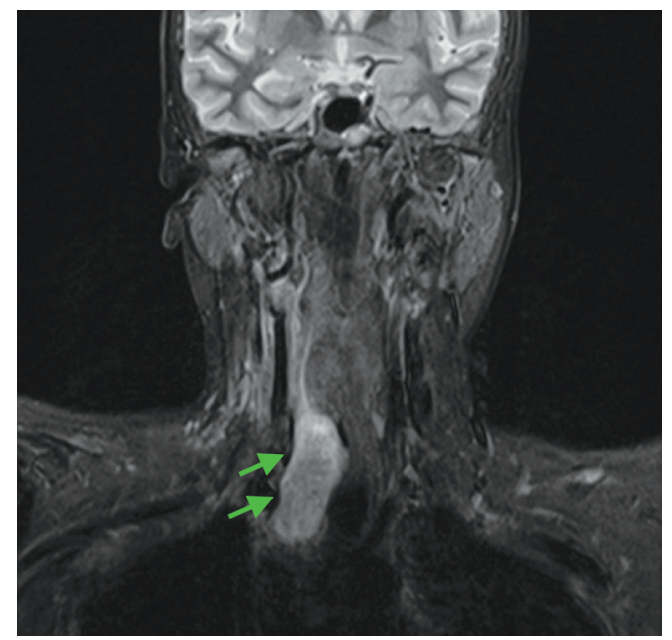

Fig. 2: MRI of the neck coronal FLAIR section showing mixed signal intensity lesion at the base of neck to the right of trachea extending from anterior aspect of the right lobe of the thyroid to the suprasternal location

Table 1: Laboratory reports

\begin{tabular}{|c|c|c|c|c|c|c|c|}
\hline Test (normal value) & $\begin{array}{l}\text { Day } 1 \text { of } \\
\text { admission }\end{array}$ & $\begin{array}{l}\text { Day } 7 \text { of } \\
\text { admission }\end{array}$ & Intraoperative & $\begin{array}{l}\text { Postoperative } \\
\text { day } 1\end{array}$ & $\begin{array}{l}\text { Postoperative } \\
\text { day } 6\end{array}$ & $\begin{array}{l}\text { On follow-up } \\
\text { at } 7 \text { months }\end{array}$ & $\begin{array}{l}\text { On follow-up } \\
\text { at } 12 \text { months }\end{array}$ \\
\hline Serum calcium (8.6-10 mg/dL) & 14.8 & 11 & & 6.0 & 7.4 & 9.6 & 10.0 \\
\hline Serum albumin $(3.5-5.2 \mathrm{~g} / \mathrm{dL})$ & 2.5 & & & 2.8 & 3.2 & 4.4 & 4.5 \\
\hline Corrected calcium (mg/dL) & 16 & & & 7.0 & 7.9 & 9.2 & 9.6 \\
\hline Serum phosphorus (2.5-4.5 mg/dL) & 4.8 & 3.2 & & 2.5 & & 4.4 & 4.8 \\
\hline PTH (15-68.3 pg/mL) & $>2,500$ & 1,768 & 252 & 28 & 155 & 135 & 98 \\
\hline $25(\mathrm{OH})$ vitamin $\mathrm{D} 3(30-100 \mathrm{ng} / \mathrm{mL})$ & 55 & & & & & 26.7 & \\
\hline Serum creatinine $(0.5-1.1 \mathrm{mg} / \mathrm{dL})$ & 2.25 & 0.8 & & & & 0.9 & \\
\hline Serum amylase (28-100 U/L) & 3,981 & & & & 137 & & \\
\hline Serum lipase (13-60 U/L) & 1,962 & & & & 127 & & \\
\hline
\end{tabular}




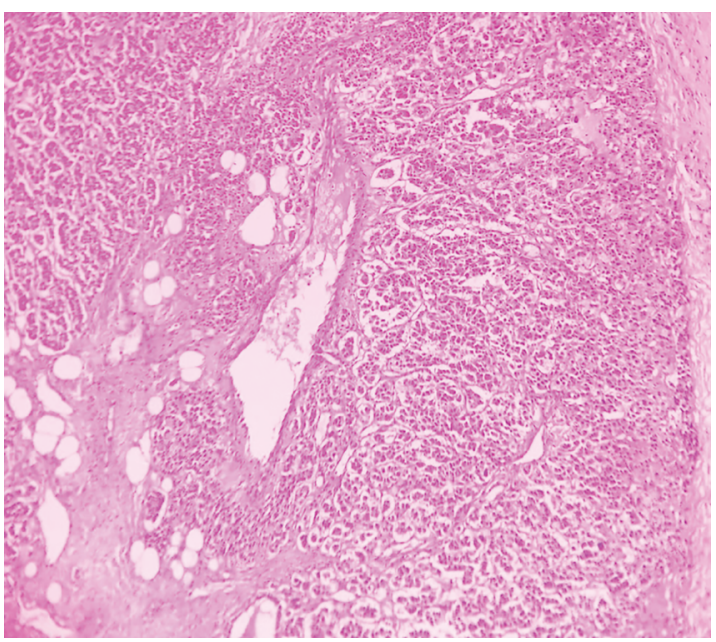

Fig. 3: Histopathology showing lesional tissue with the trabecular arrangement (arrowhead) of tumor cells with focal acinar pattern (arrow) $(H \& E \times 100)$

Hypercalcemic crisis has been reported to occur in $7 \%$ of patients presenting with severe sporadic PHPT. ${ }^{6}$ The rate of hypercalcemic crisis in parathyroid carcinoma has been reported to be $5 \%$ and has been associated with high serum PTH, calcium, and larger tumor size. ${ }^{6}$ However, atypical parathyroid adenomas also mimic parathyroid carcinoma in presentation. ${ }^{7}$ Our patient presented in hypercalcemic crisis with very high PTH levels raising the suspicion of parathyroid carcinoma. Investigations in the first antenatal visit elsewhere had shown normal unadjusted serum calcium and low $25(\mathrm{OH})$ vitamin D levels. Low vitamin D levels could have masked the hypercalcemia. ${ }^{8}$

Atypical parathyroid adenomas and carcinomas constitute $<1 \%$ of parathyroid tumors. ${ }^{9}$ While familial parathyroid syndromes MEN1, MEN2A, and MEN3 are associated with parathyroid adenomas, hyperparathyroidism jaw tumor syndrome present with atypical parathyroid adenomas, and carcinomas.. Atypical parathyroid adenomas represent a group of intermediate forms of parathyroid neoplasms of uncertain malignant potential and show atypical histological features, presenting a challenge for the differential diagnosis with parathyroid carcinomas. Clinically, they cannot be distinguished from parathyroid carcinoma preoperatively. The presence of two or more features of incomplete invasion of the capsule, fibrous bands, pronounced trabecular growth, mitotic activity $>1 / 10 \mathrm{HPF}$, tumor necrosis without unequivocal signs of capsular or vascular invasion favor the diagnosis of atypical parathyroid adenoma. ${ }^{10}$

On histopathological examination, the atypical findings of this case were cells arranged in a trabecular pattern, with few bizarre appearing and multinucleated cells without capsular invasion (Figs 3 and 4). As per the literature, ours is the second case of atypical parathyroid adenoma diagnosed and treated in pregnancy. ${ }^{11}$

The management of PHPT in pregnancy is challenging and includes the initial bridging phase, which comprises medical management, fetal monitoring, preparation for surgery, and later definitive surgery in the second trimester. ${ }^{12}$ The preoperative workup includes ultrasound and MRI examination of the neck for localization and to assess the extent of the lesion. Though a low dose sestamibi scan $(<5 \mathrm{mGy})$ is unlikely to cause fetal harm, the low sensitivity of the test does not outweigh the risk of potential

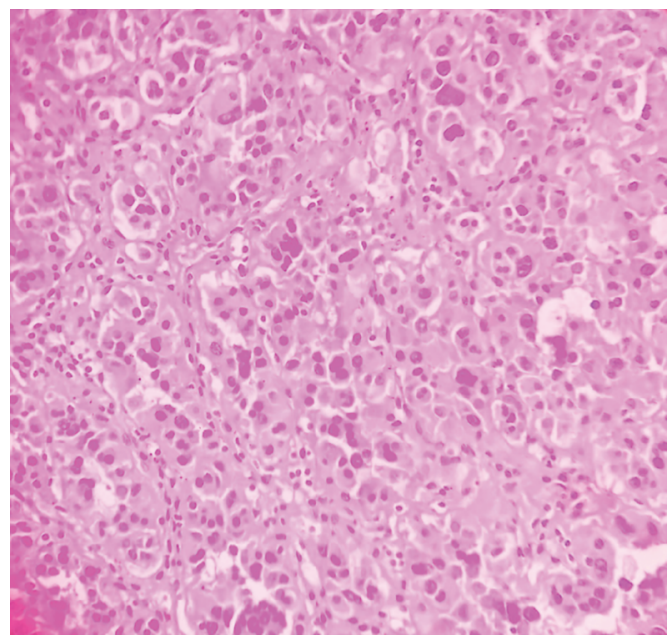

Fig. 4: Histopathology showing foci of nuclear atypia seen in the tumor $(\mathrm{H} \& \mathrm{E} \times 45)$

fetal harm. ${ }^{12}$ Four-dimensional computed tomography (4D CT) with four times higher radiation dose, compared with sestamibi is contraindicated in pregnancy. ${ }^{12}$

The choice of therapy depends on the severity of hypercalcemia. Mild hypercalcemia ( $<1 \mathrm{mg} / \mathrm{dL}$ above the upper limit of normal) may be treated with oral hydration and weekly calcium measurement. Parathyroid surgery is indicated for severe hypercalcemia as medical management options are very limited. Biphosphonates are contraindicated in pregnancy, as they cross the placenta and long-term effects on the fetal skeleton are not known. Cinacalcet, a calcium-sensing receptor (CaSR) agonist crosses the placenta and might affect placental calcium transport and development of the fetus, owing to the presence of CaSR on many tissues other than the parathyroid gland. ${ }^{12}$ Calcitonin, not known to cross the placenta can be used in hypercalcemic crisis as a bridging therapy to surgery. ${ }^{12}$ Even though hemodialysis is risky in pregnancy, it is indicated for the management of the hypercalcemic crisis. Higher incidence of stillbirth/perinatal death, lower mean gestational age at delivery, and lower birth weight were observed in women with AKI. ${ }^{13}$ A review of case studies and registries over 20 years has not shown any adverse maternal and fetal outcomes or preterm delivery due to parathyroid surgery in pregnancy. ${ }^{12}$

On further follow-up after 1 year, our patient has remained eucalcemic with mild elevated serum PTH. This is not unusual as one-third of patients undergoing parathyroidectomy will continue to have elevated PTH, despite normalization of serum calcium. ${ }^{14}$

\section{ConClusion}

Hypercalcemia is often unrecognized in pregnancy owing to the non-specific symptoms. The most common cause of hypercalcemia in pregnancy is PHPT. A high index of suspicion is needed, as undiagnosed hypercalcemia will lead to significant maternal and fetal complications. Our case highlights that effective medical management of hypercalcemic crisis as a bridging therapy to definitive surgery will result in a favorable outcome.

\section{Clinical Significance}

Effective medical management as a bridging therapy to surgery of PHPT in pregnancy will lead to better outcomes. Our case 
emphasizes the importance of vitamin D status in the presentation of PHPT and the benign entity of persistently elevated PTH levels after parathyroid surgery, even despite achieving and maintaining normal serum calcium levels.

\section{References}

1. Hu Y, Cui M, Sun Z, et al. Clinical presentation, management, and outcomes of primary hyperparathyroidism during pregnancy. Int J Endocrinol 2017;2017:3947423. DOI: 10.1155/2017/3947423.

2. Som M, Stroup JS. Primary hyperparathyroidism and pregnancy. In: Baylor University medical center proceedings. Taylor \& Francis; 2011. vol. 24 , No. 3 pp. $220-223$.

3. Ameerudden $\mathrm{S}, \mathrm{He} \mathrm{X}$. Management and surgical treatment of parathyroid crisis secondary to parathyroid tumors: report of four cases. Int Med Case Rep J 2011;4:59. DOI: 10.2147/IMCRJ. S23764.

4. Hirsch D, Kopel V, Nadler V, et al. Pregnancy outcomes in women with primary hyperparathyroidism. J Clin Endocrinol Metabol 2015;100(5):2115-2122. DOI: 10.1210/jc.2015-1110.

5. Schnatz PF, Curry SL. Primary hyperparathyroidism in pregnancy: evidence-based management. Obstetr Gynecolog Sur 2002;57(6): 365-376. DOI: 10.1097/00006254-200206000-00022.

6. Cannon J, Lew Jl, Solórzano CC. Parathyroidectomy for hypercalcemic crisis: 40 years' experience and long-term outcomes. Surgery 2010;148(4):807-812. DOI: 10.1016/j.surg.2010.07.041.

7. Mishra A, Newman D. An interesting case of life-threatening hypercalcemia secondary to atypical parathyroid adenoma versus parathyroid carcinoma. Case Rep Med 2014;2014:473814. DOI: 10.1155/2014/473814.

8. Bala $S$, Shah B, Rajput $P$, et al. Unmasking of primary hyperparathyroidism by vitamin D therapy. Indian J Nephrol 2015;25(6):377. DOI: 10.4103/0971-4065.157427.

9. Cetani F, Marcocci C, Torregrossa L, et al. Atypical parathyroid adenomas: challenging lesions in the differential diagnosis of endocrine tumors. Endocr Rela Cancer 2019;26(7):R441-R464. DOI: 10.1530/ERC-19-0135.

10. Ramaswamy AS, Vijitha T, Kumarguru BN, et al. Atypical parathyroid adenoma. Indian J Pathol Microbiol 2017;60(1):99. DOI: 10.4103/03774929.200055.

11. Razavi CR, Charitou M, Marzouk M. Maternal atypical parathyroid adenoma as a cause of newborn hypocalcemic tetany. Otolaryngol Head Neck Surg 2014;151(6):1084-1085. DOI: 10.1177/0194599814555850.

12. DiMarco AN, Meeran K, Christakis I, et al. Seventeen cases of primary hyperparathyroidism in pregnancy: a call for management guidelines. J Endocr Soc 2019;3(5):1009-1021. DOI: 10.1210/js.201800340.

13. Liu Y, Ma X, Zheng J, et al. Pregnancy outcomes in patients with acute kidney injury during pregnancy: a systematic review and meta-analysis. BMC Preg Childbirth 2017;17(1):235. DOI: 10.1186/ s12884-017-1402-9.

14. Caldwell M, Laux J, Clark M, et al. Persistently elevated PTH after parathyroidectomy at one year: experience in a tertiary referral center. J Clin Endocrinol Metabol 2019;104(10):4473-4480. DOI: 10.1210/jc.2019-00705. 